Analítica 2001

\title{
Validação de Estabilidade de Soluções
}

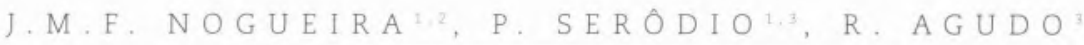

\section{Introdução}

Com as actuais exigências para produtos químicos, reagentes e soluções, nos meios laboratoriais, clínicos e de investigação, emergem como problemas importantes as questões relacionadas com os respectivos prazos de validade atribuídos [1,2]. Porque não é determinado o prazo de validade de todas as soluções e reagentes pelo fabricante quando são produzidos? Como deve ser determinada a validade de soluçōes e reagentes não especificada pelo fabricante? Como irá um laboratório estabelecer a validade de soluções e reagentes preparados internamente? Esta é pois uma preocupação crescente fundamentalmente em laboratórios farmacêuticos com sistema de qualidade implementado.

Para assegurar boas práticas de fabrico, muitos fabricantes seguem normas estabelecidas por entidades como a "International Standardization Organization" (ISO), sendo inspeccionados igualmente por auditores ISO. Alguns fabricantes são ainda obrigados a visitas periódicas do "Food and Drug Administration" (FDA), entidade que fiscaliza as empresas que comercializam produtos alimentares e farmacêuticos no mercado norte-americano. Estes fabricantes são ainda conhecidos como utilizadores das "Good Manufacturing Practices" (GMP).

Os fabricantes que são certificados pela ISO têm processos internos de controle que exigem inspecção periódica da apa- rência química e especificações dos produtos e reagentes em intervalos de tempo definidos. Os utilizadores das GMP seguem as "Standard Operating Procedures" (SOP's) exigidos pelo FDA, que recomenda que os produtos químicos da "United States Pharmacopeia" (USP) que se degradam em menos de dois anos devem possuir prazo de validade. Todos os fármacos regularizados pelo FDA e diagnósticos in-vitro requerem prazo de validade. Pelo contrário, para as matérias primas, produtos químicos e reagentes para uso laboratorial na maioria das vezes, não é exigido qualquer prazo de validade.

É a partir dos produtos químicos e reagentes que se preparam as soluções que vão ser usadas para diversos fins. 0 tempo de conservação de um reagente ou solução pode ser definido como o período de tempo durante o qual o reagente ou solução é considerado(a) utilizável para determinada aplicação, tendo em conta todas as alterações físico-químicas.

No laboratório, a inspecção regular da aparência e qualidade dos reagentes e soluções deve ser uma prática constante de rotina. A qualidade de reagentes e soluções pode por exemplo ser afectada somente pelo contacto atmosférico, que ocorre cada vez que se abre um frasco. Muitos reagentes e soluções sofrem deterioração, alterando a aparência física que é facilmente reconhecida através da mudança de cor, liquefacção e sedimentação, motivados pela exposição ao ar, humidade entre outras influências externas. No entanto, o prazo de aplicação específica de um reagente ou solução pode terminar para um certo uso mas continuar válido para outro fim.

Perante todo este cenário, os laboratórios devem criar as suas próprias "Internal Operating Procedures" (IOP's) para substituição dos reagentes e soluções após um período de tempo específico [3]. Neste contexto, a presente contribuição visa o estudo de validaçăo de estabilidade de diversas soluções químicas correntes usadas em laboratórios analíticos.

\section{Materiais e Métodos}

No presente estudo foi usado material de laboratório calibrado e reagentes que possuiam grau analítico. Foram estudadas trinta e cinco soluções entre acídicas $\left(\mathrm{HCl}, \mathrm{H}_{2} \mathrm{SO}_{4}\right.$ e $\left.\mathrm{HClO}_{4}\right)$, básicas $\left(\mathrm{NaOH}\right.$ e $\mathrm{NH}_{3}$ ), complexantes (EDTA), argentimétricas $\left(\mathrm{AgNO}_{3}\right.$ e $\left.\mathrm{NH}_{4} \mathrm{SCN}\right)$, redutoras $\left(\mathrm{Na}_{2} \mathrm{~S}_{2} \mathrm{O}_{3}\right)$, oxidantes $\left(\mathrm{I}_{2}\right.$ e $\left.\mathrm{KMnO}_{4}\right)$, sais $\left(\mathrm{NH}_{4} \mathrm{CH}_{3} \mathrm{COO},\left(\mathrm{NH}_{4}\right)_{2}\right.$

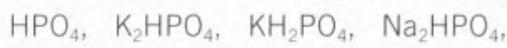
$\mathrm{NaH}_{2} \mathrm{PO}_{4}, \mathrm{NaCl}$ e $\mathrm{Na}_{2} \mathrm{SO}_{4}$ ) e eluentes para cromatografia líquida $\left(\mathrm{CH}_{3} \mathrm{OH} / \mathrm{H}_{2} \mathrm{O}\right.$, $\mathrm{CH}_{3} \mathrm{CH}_{2} \mathrm{OH} / \mathrm{H}_{2} \mathrm{O}$ e $\mathrm{CH}_{3} \mathrm{CN} / \mathrm{H}_{2} \mathrm{O}$ ), possuindo em alguns casos diversas concentrações. Os títulos foram avaliados por métodos volumétricos (ácidos, bases, complexantes, argentimétricos, redutores e oxidantes), cromatográficos (sais) e de Karl-Fisher (eluentes), validados de acordo com o recomendado

\footnotetext{
${ }^{1}$ DQB/FCUL, Campo Grande Ed. C8 - 3. ${ }^{\circ}$ Piso, 1749-016 Lisboa, Portugal; nogueira@fc.ul.pt ${ }^{2}$ CCMM, Campo Grande Ed. C8 - 4. . Piso, 1749-016 Lisboa, Portugal

${ }^{3}$ HOVIONE SA, Sete Casas, 2674-506 Loures, Portugal
} 
figura 1 Diagrama reproduzindo o período de tempo a partir do qual as soluçôes estudadas, constituidas por ácidos, bases, complexantes, argentimétricos, redutores e oxidantes, perdem estabilidade [10] figura 2 Diagrama reproduzindo o período de tempo a partir do qual as soluçōes estudadas, constituidas por sais e eluentes, perdem estabilidade [10].

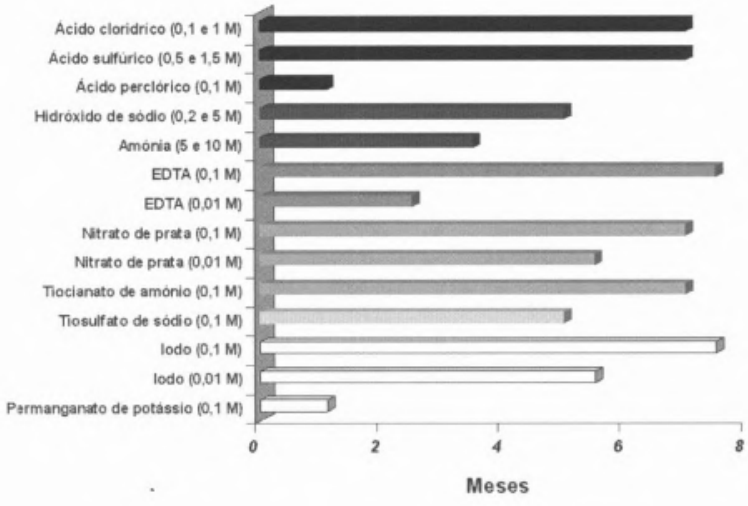

- Ácidos $=$ Bases $=$ Complexantes $=$ Argentimétricos $=$ Redutores $\square$ Oxidantes

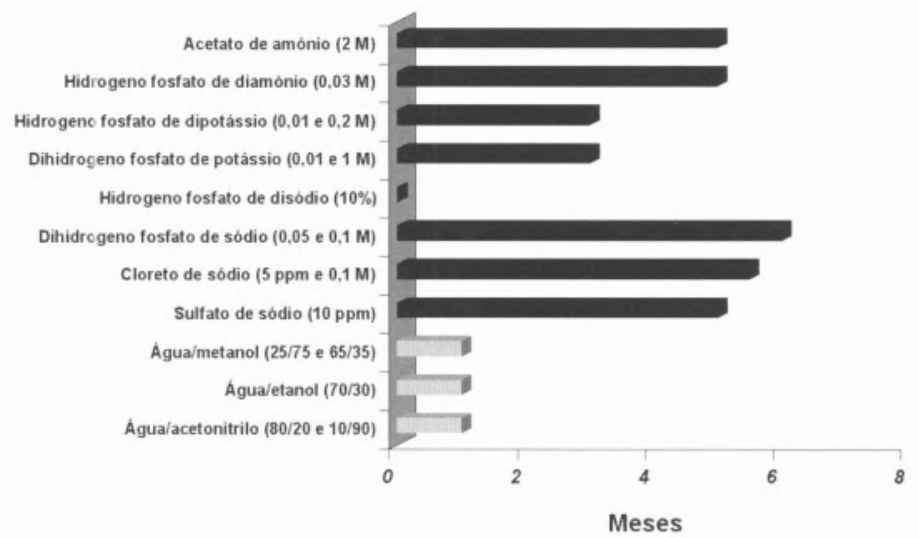

- Sais Eluentes pelas farmacopeias europeia (EP), americana (USP), inglesa (BP) e/ou segundo métodos internos [4-10]. As incertezas associados aos métodos volumétricos (< $0,25 \%)$, cromatográficos $(<0,60 \%)$ e de Karl-Fisher $(<0,15 \%)$ implementados foram estimados em sintonia com o guia EURACHEM/CITAC [11].

Durante o condicionamento das soluções a abertura dos frascos foi apenas efectuada aquando de cada análise e o menor número de vezes possível. Todos os frascos foram guardados num armário fechado, protegido da luz e à temperatura do laboratório $\left(20^{\circ} \mathrm{C}\right)$.

Estabeleceu-se uma periodicidade regular de análise das soluções, semanal durante 0 primeiro mês e posteriormente mensal, durante sete meses e meio.
Após a padronização das soluções e determinada a concentração inicial, estabeleceu-se como critério de aceitação a variação de $\pm 2 \%$ relativamente ao valor encontrado em cada solução estudada em triplicado. Sempre que a solução no decorrer do estudo de estabilidade apresentasse sinais de degradação, pela presença de partículas em suspensão, alteração de cor ou sedimentação, recorria-se à respectiva filtração. Caso aqueles sinais permanecessem, era terminado o estudo, sendo a solução rejeitada e tomado o período até então decorrido como indicador da estabilidade da solução.

\section{Resultados e Discussão}

Na generalidade, os departamentos de controle de qualidade formulam procedimentos internos específicos e relativos à tentativa de validação de reagentes e soluções químicas usadas nos vários laboratórios. Os prazos são genericamente estipulados com base na experiência adquirida pelos analistas e apenas verificados tendo em conta o comportamento dos reagentes e soluções ao longo do tempo, carecendo, no entanto, de comprovação. 0 simples uso de uma solução cujo título não seja o correcto, pode desta forma afectar toda a validação dos métodos analíticos envolvidos, colocando em risco o eficiente controlo dos fármacos e como consequência a saúde pública. 
A figura 1 reproduz o prazo de validade encontrado para as soluções estudadas continuamente, constituídas por ácidos, bases, complexantes, argentimétricos, redutores e oxidantes. Observa-se claramente que seis soluções são estáveis acima de seis meses $\left(\mathrm{HCl}, \mathrm{H}_{2} \mathrm{SO}_{4}\right.$, EDTA $0,1 \mathrm{M}), \mathrm{AgNO}_{3}(0,1 \mathrm{M}) \mathrm{NH}_{4} \mathrm{SCN}$ e I $(0,1$ $\mathrm{M})$ ), cinco acima de três meses $(\mathrm{NaOH}$, $\mathrm{NH}_{4}, \mathrm{AgNO}_{3}(0,01 \mathrm{M}), \mathrm{Na}_{2} \mathrm{~S}_{2} \mathrm{O}_{3}$ e I $(0,01 M))$ e três abaixo de três meses $\left(\mathrm{HClO}_{4}, \operatorname{EDTA}(0,01 \mathrm{M})\right.$ e $\left.\mathrm{KMnO}_{4}\right)$. Verifica-se igualmente que para soluções contendo o mesmo analito mas diferente título, a de menor concentração é geralmente menos estável, evidenciando assim maior vulnerabilidade a factores externos. As soluções em iodo $(0,1 \mathrm{M})$ e hidróxido de sódio, surpreendentemente, apresentaram estabilidade superior a seis e cinco meses.

A figura 2 reproduz o prazo de validade encontrado para diversas soluções de sais e eluentes. Verifica-se neste caso que nenhuma destas soluções é estável acima de seis meses, sendo cinco estáveis acima dos quatro meses $\left(\mathrm{NH}_{4} \mathrm{CH}_{3} \mathrm{COO},\left(\mathrm{NH}_{4}\right)_{2} \mathrm{HPO}_{4}, \mathrm{NaH}_{2} \mathrm{PO}_{4}\right.$, $\mathrm{NaCl}$ e $\mathrm{Na}_{2} \mathrm{SO}_{4}$ ) e duas acima de dois meses $\left(\mathrm{K}_{2} \mathrm{HPO}_{4}\right.$ e $\left.\mathrm{KH}_{2} \mathrm{PO}_{4}\right)$. Enquanto os eluentes estudados evidenciaram um período de estabilidade de apenas um mês, a solução de $\mathrm{Na}_{2} \mathrm{HPO}_{4}$ (10\%) demonstrou muito baixa estabilidade e por conseguinte, recomenda-se que a mesma seja somente usada após preparação.

0 alargar de prazo de validade de uma solução tem um impacto maior do que aparentemente se possa imaginar. Considerando uma solução que ao fim de alguns dias se torna instável ou pelo contrário possa apresentar estabilidade durante vários meses, são óbvias as vantagens inerentes a este facto. Uma solução se for preparada menos vezes num determinado periodo de tempo, permite direccionar o tempo despendido aquando da preparação e padronização, para o trabalho analítico mais importante. Também o consumo de menores quantidades de reagentes, implica obviamente custos menos onerosos no controlo da qualidade. A longo prazo, o efeito conjunto dos parâmetros tempo e custo torna-se assim decisivos, aumentando a rapidez de resposta de um laboratório para as análises que são requeridas, possibilitando desta forma melhorar igualmente a política de aquisição de reagentes que é necessário adquirir num determinado período de tempo.

A presente contribuição tem objectivo recomendatório, relativamente à estabilidade das soluções analíticas estudadas, apresentando-se como resposta antecipada às novas e crescentes exigências no controlo da qualidade que se adivinha emergir num futuro próximo. Para as indústrias farmacêuticas, é uma mais-valia relativamente à concorrência e um resguardo relativamente às permanentes auditorias, podendo considerar-se mesmo como um ponto de partida numa área ainda pouco investigada e que uma vez desenvolvida pode promover uma maior rentabilidade nos laboratórios analíticos.
Este estudo é considerado pioneiro, uma vez na grande maioria dos laboratórios químicos em geral, não haver documentação e/ou protocolos que estabeleçam prazos de validade para as soluções analíticas, devido fundamentalmente à inexistência de obrigatoriedade legal.

\section{Referências}

[1] E. Prichard, Quality in the Analytical Chemistry Laboratory, John Wiley \& Sons Ltd, UK, 1995.

[2] R.E. Lawn, M. Thompson and R.F. Walker, Proficiency Testing in Analytical Chemistry, The Royal Society of Chemistry, UK, 1997.

[3] W.E. Garner, M.S. Barge, J.P. Ussuary, Good Laboratory Practice, ACS, Washington DC, 1992.

[4] P. Serôdio, Validation of the Stability of Solutions, Report AA/RE181, Hovione SA, FCUL, 2001.

[5] United States Pharmacopoeia, Vol. XXI, 1985.

[6] United States Pharmacopoeia, Vol. XXII, 1990.

[7] United States Pharmacopoeia, Vol. XXIII, 1995.

[8] British Pharmacopoeia, A45, Vol. I, 1980.

[9] British Pharmacopoeia, Vol. I, 1988.

[10] J.M.F. Nogueira, P. Serôdio, Determination of Expiration Date of Chemical Solutions, Accreditation and Quality Assurance, 2002 (in press).

[11] Quantifying Uncertainty in Analytical Measurement, EURACHEM/CITAC Guide, 2nd Edition, 2000.

On peut concevoir encore, que dans des mains criminelles, le radium puisse devenir très dangereux, et ici on peut se demander si l'humanité a avantage à connaître les secrets de la nature, si elle est mûre pour en profiter ou si cette connaissance ne lui sera pas nuisible. L'exemple de Nobel est charactéristique, les explosifs puissants on permis aux hommes de faire des travaux admirables. Ils sont aussi un moyen terrible de destruction entre les mains des grands criminels qui entraînent les peuples vers la guerre. Je suis de ceux qui pensent, avec Nobel, que l'humanité tirera plus de bien que de mal des découvertes nouvelles. 


\section{Subscribe Now!}

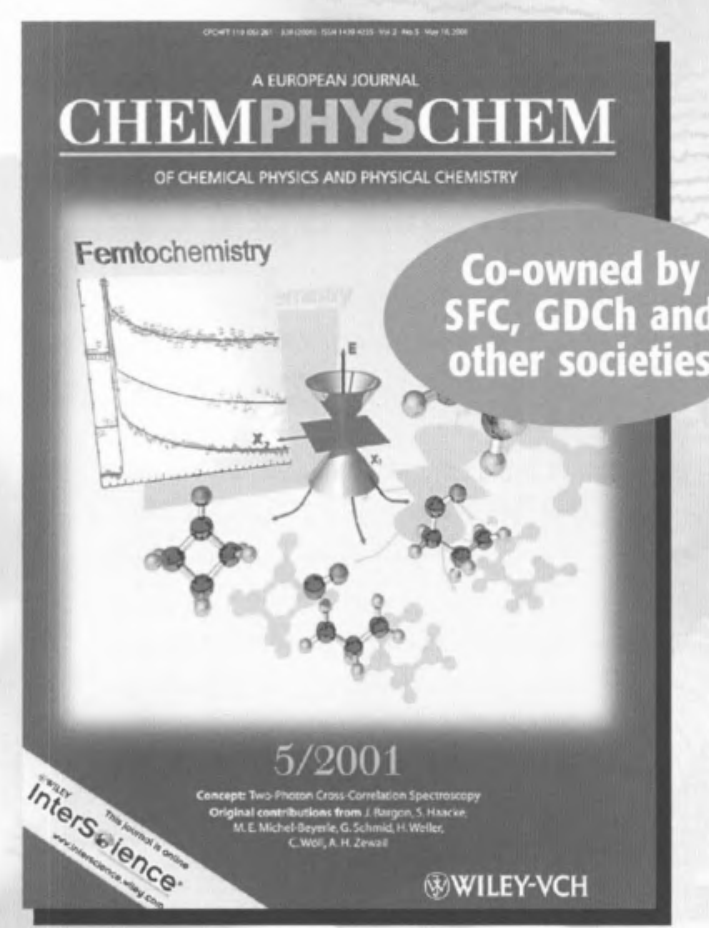

\section{ChemPhysChem}

A European Journal of Chemical Physics and Physical Chemistry

2002 Volume 3, 12 issues per year,

ISSN Print 1439-4235

ISSN Electronic 1439-7641

\section{ChemPhysChem - Where Chemistry Meets Physics Meets Chemistry....}

ChemPhysChem amalgamates the wide and flourishing field ranging - to name just a few topics -

- from atmospheric science to hard and soft condensed matter

- from femtochemistry to nanotechnology

- from complex biological systems to single molecule research

- from clusters and colloids to catalysis and surface science

- from electro- to photochemistry

\section{Papers from distinguished scientists worldwide, such as}
Z. I. Alferov
G. Ertl
C. Amatore
C. Friend
C. A. Mirkin
C. D. Bain
J. S. Kilby
C. N. R. Rao
V. Balzani
H. Kroemer
J.-M. Savéant
C. Bräuchle
R. Lavery
R. J. Saykally
E. A. Carter
J.-M. Lehn
G. Wegner
A. Corma
R. D. Levine
C. Zannoni
F. C. De Schryver
H. Matsuhara
R. N. Zare
A. H. Zewail

Available as a separate journal and as a part of attractive packages with Angewandte Chemie (Int. Ed.) Please visit www.chemphyschem.com

\section{Virtual Sample Copy: FREE online access to full text of sample copy: www.interscience.wiley.com}

Please enter my/our 2002 subscription to ChemPhysChem

\section{Please tick: $\square$ private $\square$ business}

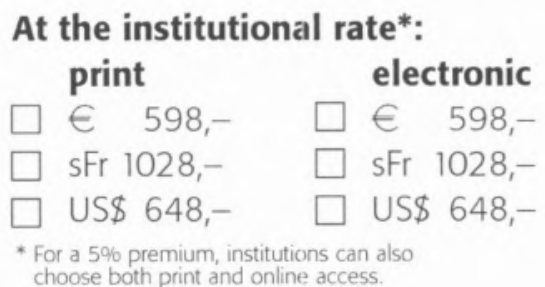

At the institutional rate*: print

$\square \in 598,-$ sFr 1028,US\$ 648,* For a $5 \%$ premium, institutions can also

electronic

$\in$ 598,-

sFr 1028,-

US\$ 648,-

$$
\text { also }
$$

At the personal member rate: print

$\square \in 148$,- $\quad$ Europe

$\square \mathrm{sFr} 248,-\quad$ Switzerland

$\square$ US\$ 158,- $\quad$ All other countries

\section{Please send me a free sample copy}

Please return this order form to your local bookseller or to:

Customers in Germany, Austria, and Switzerland:

WILEY-VCH Reader Service

P. O. Box 101161

D-69451 Weinheim, Germany

Phone: +49 (0) 6201-606 147

Fax: $+49(0) 6201-606172$

e-mail: subservice@wiley-vch.de
Customers in all other areas:

John Wiley \& Sons, Ltd.

Journals Administration Department

1 Oldlands Way

Bognor Regis West Sussex, PO22 9SA, UK

Phone: +44 (0) 1243-779 777

Fax +44 (0) $1243-843232$

e-mail:cs-journals@wileyco.uk 